\title{
A meta-analysis of the watch-and-wait strategy versus total mesorectal excision for rectal cancer exhibiting complete clinical response after neoadjuvant chemoradiotherapy
}

Guilin $\mathrm{Yu}^{1 \dagger}$, Wenqing $\mathrm{Lu}^{2 \dagger}$, Zhouguang Jiao ${ }^{3}$, Jun Qiao ${ }^{4}$, Shiyang $\mathrm{Ma}^{4}$ and Xin Liu${ }^{4^{*}}$

\begin{abstract}
Background: Some clinical researchers have reported that patients with $\mathrm{CCR}$ (clinical complete response) status after neoadjuvant chemoradiotherapy (nCRT) could adopt the watch-and-wait (W\&W) strategy. Compared with total mesorectal excision (TME) surgery, the W\&W strategy could achieve a similar overall survival. Could the W\&W strategy replace TME surgery as the main treatment option for the CCR patients? By using the meta-analysis method, we evaluated the safety and efficacy of the W\&W strategy and TME surgery for rectal cancer exhibiting CCR after nCRT.

Methods: We evaluated two treatment strategies for rectal cancer with cCR after nCRT up to July 2021 by searching the Cochrane Library, PubMed, Wanfang, and China National Knowledge Infrastructure (CNKI) databases. Clinical data for primary outcomes (local recurrence, cancer-related death and distant metastasis), and secondary outcomes (disease-free survival (DFS) and overall survival (OS)) were collected to evaluate the efficacy and safety in the two groups.
\end{abstract}

Results: We included nine studies with 818 patients in the meta-analysis, and there were five moderate-quality studies and four high-quality studies. A total of 339 patients were in the W\&W group and 479 patients were in the TME group. The local recurrence rate in the W\&W group was greater than that in the TME group in the fixed-effects model (OR $8.54,95 \% \mathrm{Cl} 3.52$ to $20.71, P<0.001$ ). The results of other outcomes were similar in the two groups.

Conclusion: The local recurrence rate of the W\&W group was greater than that in the TME group, but other results were similar in the two groups. With the help of physical examination and salvage therapy, the W\&W strategy could achieve similar treatment effects with the TME approach.

Trial registration: Protocol registration number: CRD42021244032.

Keywords: Watch-and-wait, Complete clinical response, Total mesorectal excision, Rectal cancer, Meta-analysis

*Correspondence: liuxin5626855@sina.com

${ }^{\dagger}$ Guilin Yu and Wenging Lu contributed equally to this work.

${ }^{4}$ Department of Colorectal Surgery, Cancer Hospital of China Medical University, Liaoning Cancer Hospital and Institute, No. 44 Xiaoheyan Road, Dadong District, Shenyang 110042, Liaoning Province, People's Republic of China

Full list of author information is available at the end of the article

\section{Background}

Given its high incidence and fatality rate, rectal cancer seriously endangers human health [1]. To ensure the radical resection of the tumor, some patients with low rectal cancer need to have their anus removed [2]. The major trauma creates physical and psychological problems for the patients [3]. Neoadjuvant chemoradiotherapy (nCRT) combined with surgery has become the standard 
treatment mode for the locally advanced rectal cancer. nCRT can reduce the local recurrence and tumor size in patients with locally advanced rectal cancer [4]. In addition, it can increase the chance of preserving the anus by shrinking and downgrading tumors [5]. Multiple colorectal cancer guidelines recommend TME after nCRT as the standard treatment for locally advanced rectal cancer, but TME surgery has various complications, such as bleeding, intestinal obstruction, anastomotic leakage, and other complications [6]. TME could also have a longterm negative impact on the defecation control and sexual functions $[7,8]$.

Approximately $20 \%$ rectal cancer patients could have complete tumor regression after nCRT, and the specific phenomenon is defined as clinical complete response (cCR) [9]. In 2004, the study of Habr-Gama et al. proposed that patients with $\mathrm{CCR}$ status could adopt the watch-and-wait (W\&W) treatment strategy. Since then, a series of clinical researches have promoted the discussion about the treatment strategy of $\mathrm{CCR}$ patients $[10$, 11]. Compared with TME surgery, the W\&W strategy could achieve a similar overall survival and a better preservation of organ anatomy and physiological function [12]. Some related meta-analyses had published. The results indicated that the W\&W group exhibited a higher local recurrence rate than the TME group, but the overall survival was similar in the two groups $[13,14]$. In our study, we included a larger number of studies with no difference in the baseline data (the available of clinical data pre-T stage, pre-N stage, pre-TNM stage, and preclinical stage had no significant difference, $P>0.05$ ) to assess the advantages and disadvantages between the two approaches. We compared the primary outcomes (local recurrence, cancer-related death, and distant metastasis) and secondary outcomes (DFS and OS) to evaluate the efficacy and safety in the two approaches. The results of our study provide clinical evidence for the treatment of locally advanced rectal cancer.

\section{Methods}

\section{Literature search}

Our research method has completed the protocol registration in PROSPERO (Protocol registration number: CRD42021244032; the link to the protocol: https:// www.crd.york.ac.uk/prospero/\#recordDetails). The more details were in the supplementary material 1 and 2. We performed the meta-analysis according to the PRISMA guidelines (The more details were in the supplementary material 3). The specific information of the PICOS included population, intervention, comparator and outcomes. Population: rectal cancer patients achieved cCR response after nCRT; intervention: watch-and-wait strategy; comparator: total mesorectal excision; outcomes: primary outcomes (local recurrence, cancer-related death, and distant metastasis) and secondary outcomes (DFS and OS) (The more details were in the supplementary material 4).

We performed a systematic search of the PubMed, Cochrane Library, Embase, CNKI (China National Knowledge Infrastructure), and Wanfang databases to obtain relevant literature (up to July 2021). The search string was built as follows: "watch-and-wait" or "nonoperative management" or "total mesorectal excision" or "neoadjuvant chemoradiotherapy" and "rectal cancer". Information on the search terms is shown in supplementary material 5.

\section{Inclusion and exclusion criteria}

The study had four inclusion criteria: the study had consistent baseline database and the available clinical data between the two groups (the available clinical data pre- $\mathrm{T}$ stage, pre-N stage, pre-TNM stage and pre-clinical stage had no significant difference, $P>0.05$ ); total mesorectal excision included abdominal-perineal resection (APR), Dixon and other radical surgical approaches, but not local excision; rectal cancer patients (stage I to III) with cCR status after nCRT and randomized Controlled Trial (RCTs), retrospective comparative non-randomized studies (RCNTs), prospective comparative non-randomized studies (PCNTs), cohort studies, or case-control studies. The details of neoadjuvant treatment and the diagnostic criteria of each method in each study are shown in supplementary material 6 and 7 . The exclusion criteria were as follows: a significant difference in baseline data and no valuable information of the study; as well as rectal cancer patients who did not achieve CCR status after nCRT; and reviews, case reports, or other unsuitable types.

\section{Data extraction and quality control}

The clinically useful data were collected by two reviewers independently according to the Newcastle-Ottawa Scale (NOS) guidelines [15]. Any disagreement was resolved by discussion until consensus was reached or consulted by a third author. XL, ZGJ, and SYM performed the literature search and collected the data. We conducted a preliminary sorting of the referenced documents to exclude duplicate documents. Then, we read the title and abstract of the article, and excluded studies with no control group, no rectal cancer, and incomplete data. Finally, the full text was read and the final documents were screened out. The details of the collected data are listed in the tables. Table 1 mainly contains basic information, such as: the first author, publication data, author's country, patient age, mean tumor diameter, mean distance from the anal verge, study size and study type. Table 2 contains clinical stage, $\mathrm{T}$ stage, $\mathrm{N}$ 


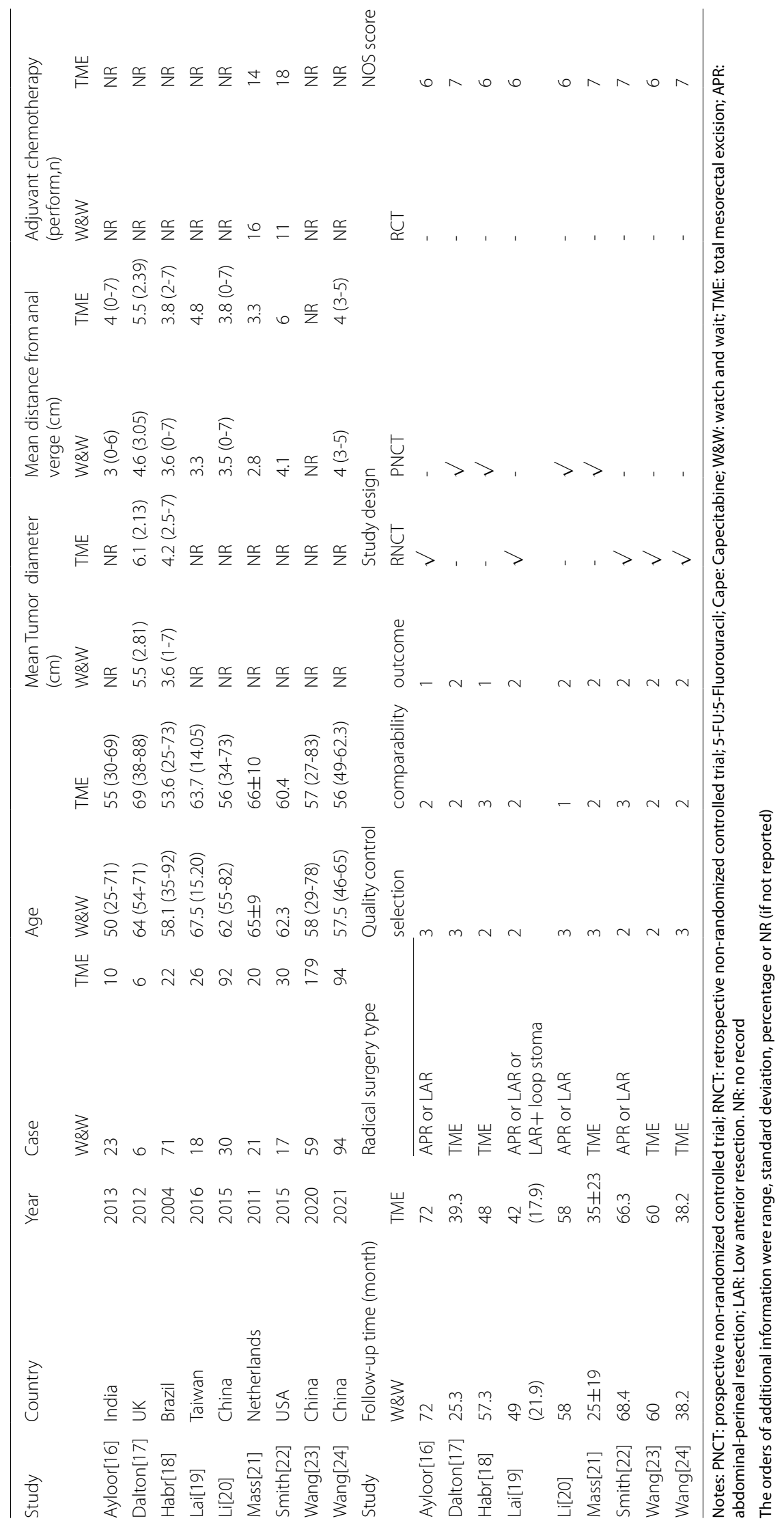


Table 2 clinical stage, T stage and N stage and Pathlologic T stage of the included articles

\begin{tabular}{|c|c|c|c|c|c|c|c|c|c|c|c|c|c|c|c|}
\hline \multirow[t]{3}{*}{ Study } & \multicolumn{15}{|c|}{ Clinical stage } \\
\hline & \multicolumn{4}{|l|}{ । } & \multicolumn{4}{|l|}{$\|$} & \multicolumn{5}{|l|}{ III } & \multicolumn{2}{|l|}{ IV } \\
\hline & \multicolumn{2}{|l|}{ W\&W } & \multicolumn{2}{|l|}{ TME } & \multicolumn{2}{|l|}{ W\&W } & \multicolumn{2}{|l|}{ TME } & \multicolumn{2}{|l|}{$W \& W$} & \multicolumn{3}{|l|}{ TME } & W\&W & TME \\
\hline Ayloor[17] & \multicolumn{2}{|l|}{$N R$} & \multicolumn{2}{|l|}{ NR } & \multicolumn{2}{|l|}{$N R$} & \multicolumn{2}{|l|}{ NR } & \multicolumn{2}{|l|}{ NR } & \multicolumn{3}{|l|}{ NR } & NR & NR \\
\hline Dalton[18] & \multicolumn{2}{|l|}{ NR } & \multicolumn{2}{|l|}{$N R$} & \multicolumn{2}{|l|}{ NR } & \multicolumn{2}{|l|}{ NR } & \multicolumn{2}{|l|}{ NR } & \multicolumn{3}{|l|}{ NR } & NR & NR \\
\hline Habr[19] & \multicolumn{2}{|l|}{ NR } & \multicolumn{2}{|l|}{ NR } & \multicolumn{2}{|l|}{ NR } & \multicolumn{2}{|l|}{ NR } & \multicolumn{2}{|l|}{ NR } & $N R$ & & & NR & NR \\
\hline Lai[20] & NR & & NR & & 11 & & 8 & & 7 & & 18 & & & NR & NR \\
\hline Li[22] & NR & & NR & & $N R$ & & NR & & $N R$ & & $N R$ & & & NR & NR \\
\hline Mass[23] & $N R$ & & NR & & NR & & NR & & NR & & $N R$ & & & NR & NR \\
\hline Smith[26] & 1 & & 2 & & 10 & & 16 & & 6 & & 12 & & & 0 & 0 \\
\hline Wang[28] & 8 & & 2 & & 17 & & 26 & & 25 & & 49 & & & NR & NR \\
\hline Wang[28] & NR & & NR & & NR & & NR & & NR & & NR & & & NR & NR \\
\hline Study & T stage & & & & N stag & & & & Pathlo & gic Tst & & & & Pathlol & V stage \\
\hline & $\mathrm{T} 1-\mathrm{T} 2$ & & T3-T4 & & No & & $\mathrm{N} 1-\mathrm{N} 2$ & & & & & & & & \\
\hline & W\&W & TME & W\&W & TME & W\&W & TME & W\&W & TME & урт0 & ypT1 & ypT2 & урT3 & ypT4 & ypNo & ypN1-N2 \\
\hline Ayloor[16] & 9 & 4 & 14 & 6 & NR & NR & NR & NR & 6 & NR & 3 & 1 & NR & 6 & 4 \\
\hline Dalton[17] & 1 & $N R$ & 5 & 6 & 1 & NR & 5 & 6 & NR & NR & $N R$ & $N R$ & NR & NR & NR \\
\hline Habr[18] & 14 & 1 & 57 & 21 & 55 & 16 & 16 & 6 & $N R$ & NR & $N R$ & $N R$ & NR & NR & $N R$ \\
\hline Lai[19] & NR & NR & NR & $N R$ & NR & NR & NR & NR & NR & NR & NR & NR & NR & NR & NR \\
\hline Li[20] & 8 & 24 & 22 & 68 & 14 & 39 & 16 & 53 & NR & NR & $N R$ & NR & NR & NR & NR \\
\hline Mass[21] & 6 & 1 & 15 & 19 & 6 & 3 & 15 & 17 & NR & NR & $N R$ & $N R$ & $N R$ & $N R$ & NR \\
\hline Smith[22] & 2 & 4 & 15 & 26 & 11 & 18 & 6 & 12 & $N R$ & NR & $N R$ & $N R$ & $N R$ & $N R$ & $N R$ \\
\hline Wang[23] & 6 & 8 & 53 & 171 & 14 & 47 & 45 & 132 & $N R$ & NR & $N R$ & $N R$ & NR & NR & NR \\
\hline Wang[24] & 9 & 8 & 85 & 86 & 19 & 24 & 75 & 70 & NR & NR & NR & NR & NR & NR & $N R$ \\
\hline
\end{tabular}

NR:no record

stage and the clinical staging after nCRT. Table 3 contains long-term outcomes of the patients. The primary and secondary outcomes are mainly shown in Table 3. LE, CRD and DM were the primary outcomes of the study, whereas 2-year, 3-year, and 5-year DFS and OS were the secondary outcomes. The other information of basic information are shown in supplementary material 8. The details of salvage therapy in the W\&W group are shown in supplementary material 9 . We tried to use various methods to obtain more missing data, but we failed to acquire more valuable data.

\section{Quality assessment}

We assessed the qualities of the included studies by using the NOS assessment scale (Newcastle-Ottawa Quality Assessment Scale). Study qualities were classified as high level $(7 \leq$ scores $\leq 9)$, middle level $(4<$ scores $\leq 6)$, and low level $(1<$ scores $\leq 3)$. We included five moderatequality studies and four high-quality studies, and the nine included studies were five RCNT and four PNCT. We did not find any RCTs through a literature search. The details are shown in Table 1.

\section{Statistical analysis}

We performed the meta-analysis by using RevMan 5.0 and Stata 11.0 software. Continuous data and dichotomous data were evaluated by the standardized mean differences (SMDs) and relative risks (ORs or RRs) with 95\% confidence intervals respectively. We used the $I^{2}$ statistic and funnel plots to assess the heterogeneity and publication bias separately. We used random effects models to analyze the data with huge heterogeneity $\left(I^{2} \geqq 50 \%\right)$ and the fixed-effects model for little heterogeneity $\left(I^{2}<50 \%\right)$.

\section{Results}

\section{Study selection}

After we completed the relevant search, we deleted duplicate studies $(N=94354)$, as well as studies with insufficient data $(N=3565)$, no control group $(N=15092)$, no rectal cancer $(N=11471)$, not-cCR $(N=3223)$, and not-W\&W $(N=2886)$ (Fig. 1$)$. After the literature screening, a total of nine studies with 818 patients were included [16-24]. A total of 339 patients were in the W\&W group, and 479 patients were in the TME group. The meta-analysis included five Eastern studies and four Western studies. Eight suitable English studies and one suitable Chinese study (Wang ${ }^{23}$ ) were identified. 


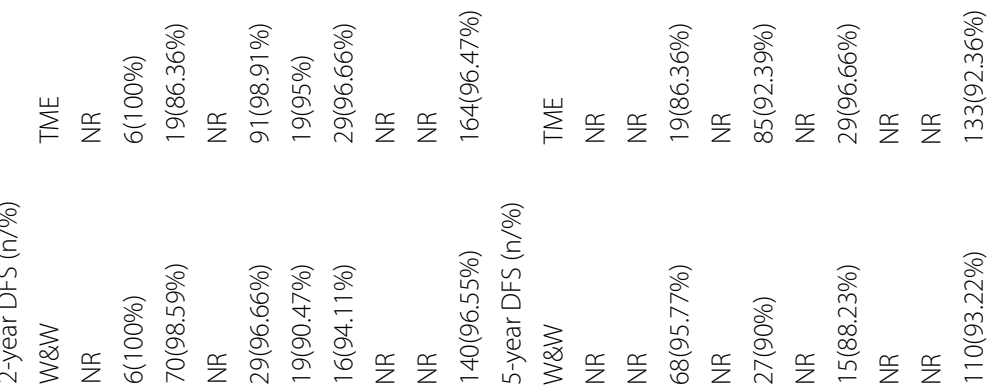

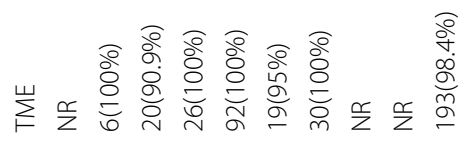

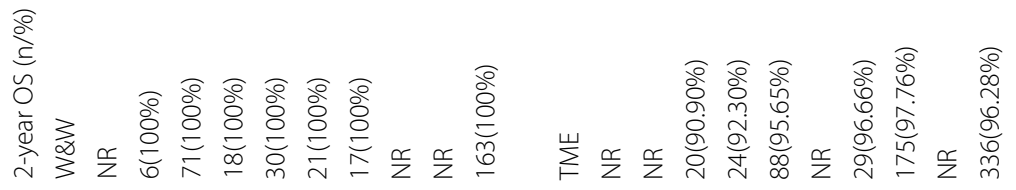

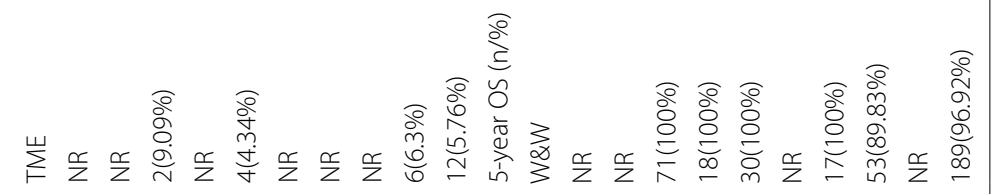

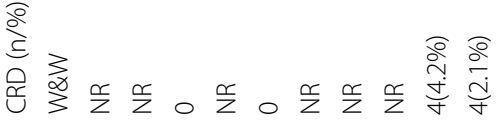

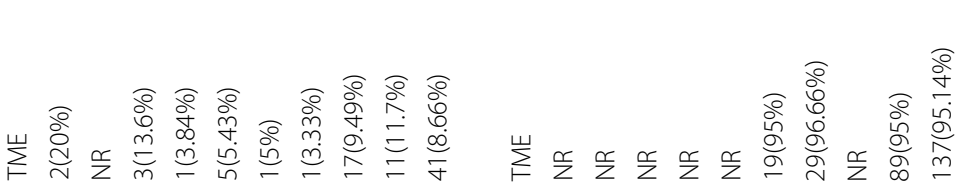

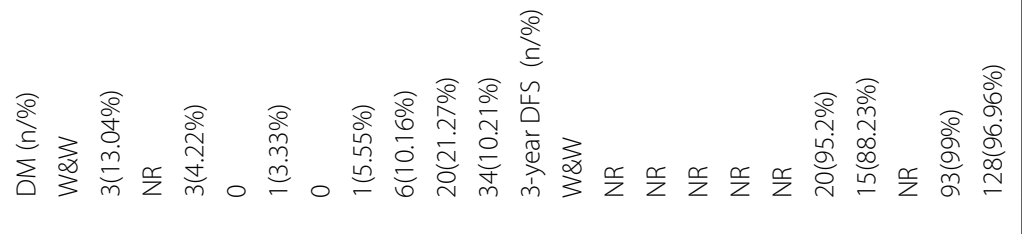

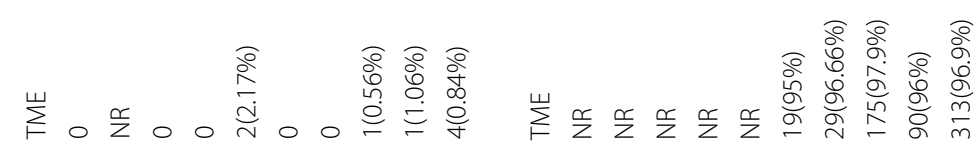

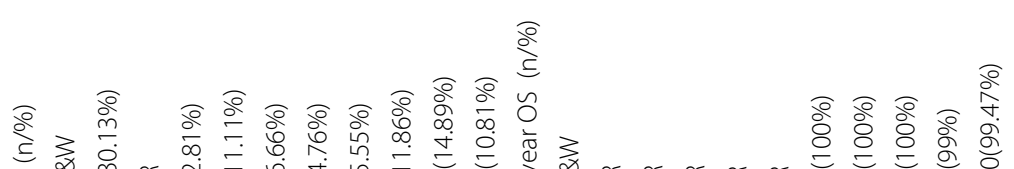

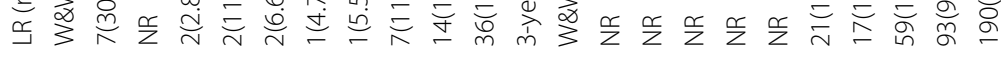

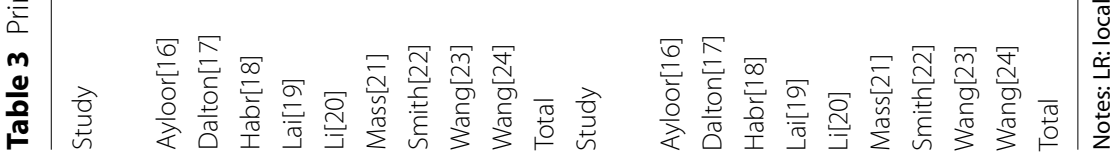




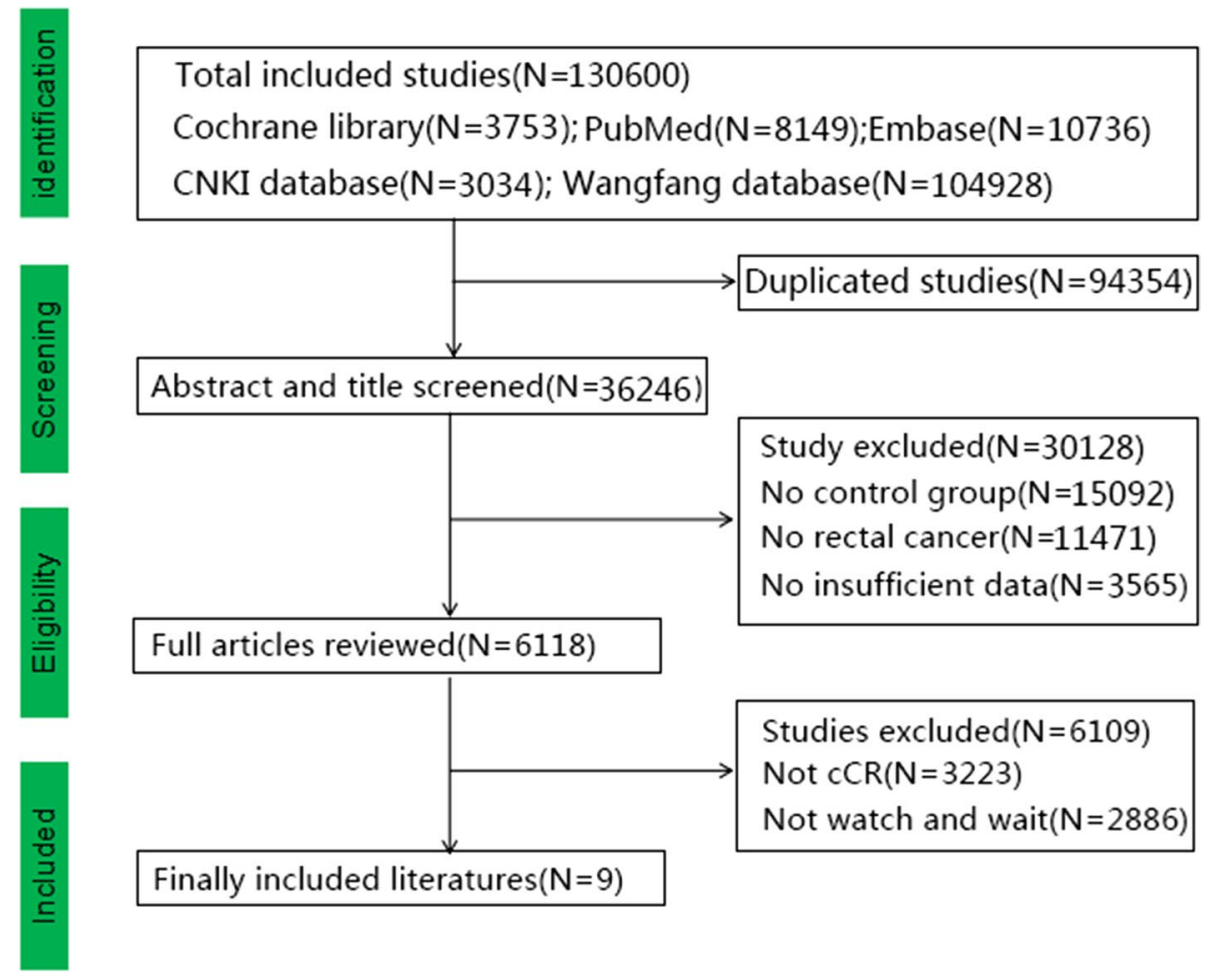

Fig. 1 Flowchart of the included studies

Statistical methods used in the included studies were $\chi^{2}$ and $t$ tests. Ayloor et al. [16] and Dalton et al. [17] did not describe statistical methods in the article. Baseline characteristics of other studies have been statistically analyzed, and there was no significant differences in the baseline characteristics (such as pretreatment $\mathrm{T}$ stage, $\mathrm{N}$ stage, TNM stage) [25-29]. The study of Smith et al. [22] included one stage IV patient. We excluded the stage IV patients and screened valuable data for further research. The details of the included studies are shown in Table 1 and supplementary material 8 . A total of $86.4 \%$ of patients with T3-4 stage disease and $63.9 \%$ of patients with N1-2 stage disease were included before nCRT (Table 2). Only the study of Ayloors et al. [16] reported the clinical stage after nCRT, and most tumors were clinically downgraded. We hypothesized that the majority of the patients had tumor downstaging after nCRT (Table 2). The details of long-term outcomes (local recurrence, distant metastasis, cancer-related death, disease-free survival, and overall survival) are shown in Table 3 . The details of salvage therapy and more information on the $\mathrm{W} \& \mathrm{~W}$ group are shown in supplementary material 10 and 11 . A total of 36 patients had local recurrence in the W\&W group and 30 (83.33\%) underwent salvage therapy. TME surgery was the main type of salvage therapy for local recurrence. Twelve (40\%) patients underwent APR surgery, and the overall survival times in the Ayloor and Li studies were 66 and 49.5 months, respectively.

\section{W\&W group versus the TME group Primary outcomes}

Local recurrence, distant metastasis, and cancer-related death Clinical data of the local recurrence rate were reported in 8 studies, and the local recurrence rate of the W\&W group was greater than that of the TME group in the fixed-effects model (OR 8.54, 95\% CI 3.52 to 20.71 , $P<0.001, X^{2}=3.80, P=0.80, I^{2}=0 \%, 10.81 \%$ vs $0.84 \%$, Fig. 2a). The distant metastasis rate was similar in the W\&W group and the TME group in the fixed-effects model with little heterogeneity (OR 1.12, 95\% CI 0.68 to $1.84, P=0.67, X^{2}=6.51, P=0.48, I^{2}=0 \%, 10.21 \%$ vs 8.66\%, Fig. 2b). Cancer-related death (OR 0.40, 95\% CI 0.14 to $1.15, P=0.35, \chi^{2}=2.10, P=0.35, I^{2}=5 \%, 2.05 \%$ vs $5.76 \%$, Fig. $2 \mathrm{c}$ ) was similar between the two groups in the fixed-effects model with high heterogeneity.

\section{Secondary outcomes 2-year OS, 2-year DFS, 3-year OS, 3-year} DFS, 5-year OS, and 5-year DFS

The clinical data of 2-year OS were reported in 6 studies, the W\&W group and the TME group had similar 


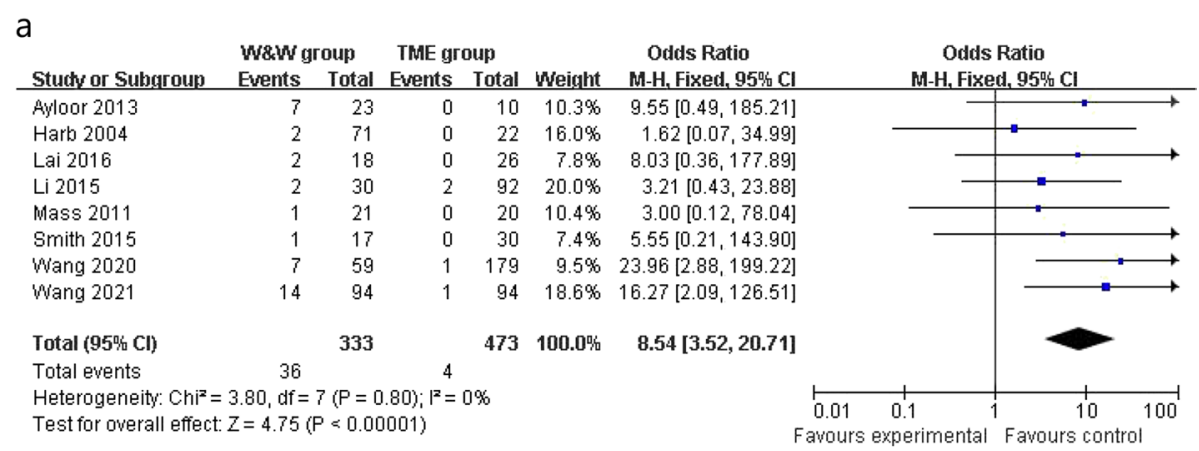

b

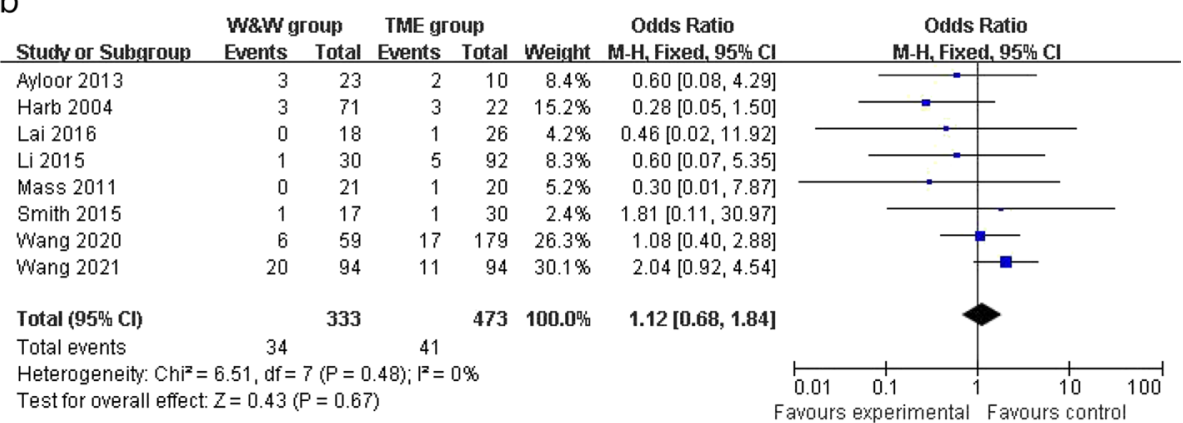

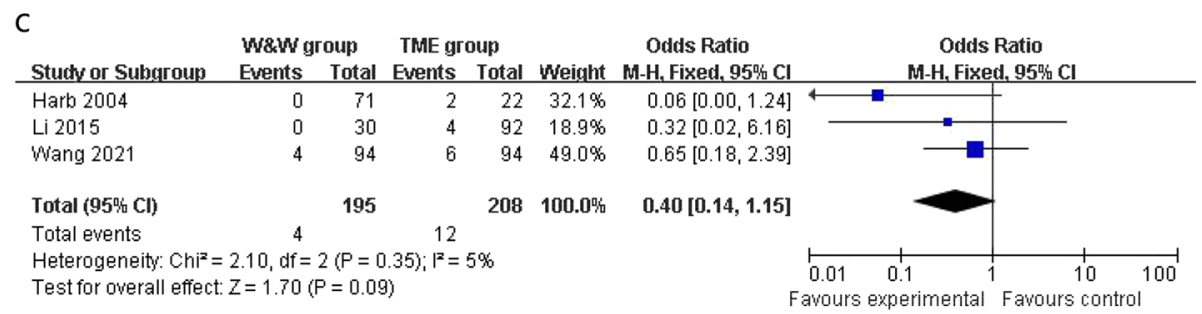

Fig. 2 Outcomes of W\&W group versus TME group. a Local recurrence. b Distant metastasis. c Cancer-related death

2-year OS in the fixed-effects model with minimal heterogeneity (OR $3.65,95 \%$ CI 0.89 to $15.05, P=0.07, \chi^{2}=$ $1.71, P=0.79, I^{2}=0 \%, 100.00 \%$ vs $98.43 \%$, Fig. 3a). Five studies reported 2-year DFS and the two groups had similar 2-year DFS in the fixed-effects model with minimal heterogeneity (OR $1.93,95 \% \mathrm{CI} 0.59$ to $6.37, P=0.28$, $X^{2}=4.18, P=0.24, I^{2}=28 \%, 96.55 \%$ vs $96.47 \%$, Fig. $\left.3 \mathrm{~b}\right)$. Three-year OS (OR 3.19, 95\% CI 0.78 to $12.98, P=0.10$, $X^{2}=0.18, P=0.98, I^{2}=0 \%, 99.47 \%$ vs $96.90 \%$, Fig. 3 c) and 3-year DFS (OR 1.51, 95\% CI 0.44 to $5.13, P=0.51$, $X^{2}=3.27, P=0.20, I^{2}=39 \%, 96.96 \%$ vs $95.14 \%$, Fig. $3 \mathrm{~d}$ ) were similar in both groups in the fixed-effects model with minimal heterogeneity. In addition, 5-year OS (OR $1.79,95 \%$ CI 0.27 to $11.80, P=0.54, X^{2}=10.50, P=0.03$, $I^{2}=62 \%, 96.92 \%$ vs $96.28 \%$, Fig. 3e) was similar in two groups in the random-effects model with significant heterogeneity, whereas 5 -year DFS (OR 1.03, 95\% CI 0.39 to $2.75, P=0.95, \chi^{2}=3.51, P=0.17, I^{2}=43 \%, 93.22 \%$ vs
92.36\%, Fig. 3f) was similar in two groups in the fixedeffects model with minimal heterogeneity.

\section{Publication bias}

By using RevMan 5.0 and stata11.0 software, we used clinical recurrence data to detect publication bias. We obtained the funnel plot with the distributed points in the funnel plot. And the results of Egger's test and Begg's test indicated, there was no statistical difference for publication bias $(P>0.05)$. The details are shown in supplementary material 12 .

\section{Discussion}

According to NCCN guidelines, nCRT is the standard treatment for locally advanced rectal cancer. In 2004, Professor Habr-Gama first proposed the non-operative management (watch-and-wait strategy) for rectal cancer patients with a cCR response [30]. In 2012, Professor Mass proposed five diagnostic criteria for $\mathrm{CCR}$. The 
a

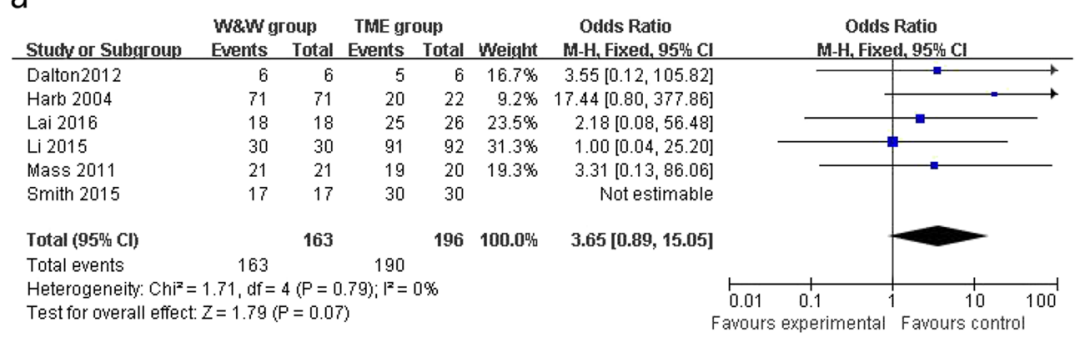

b

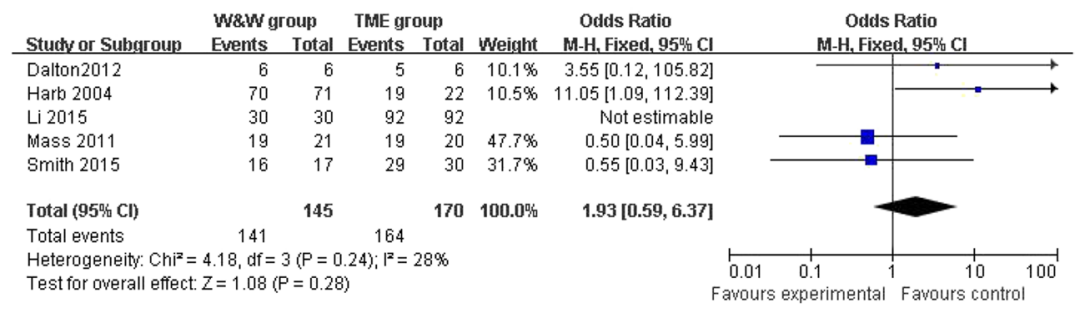

C

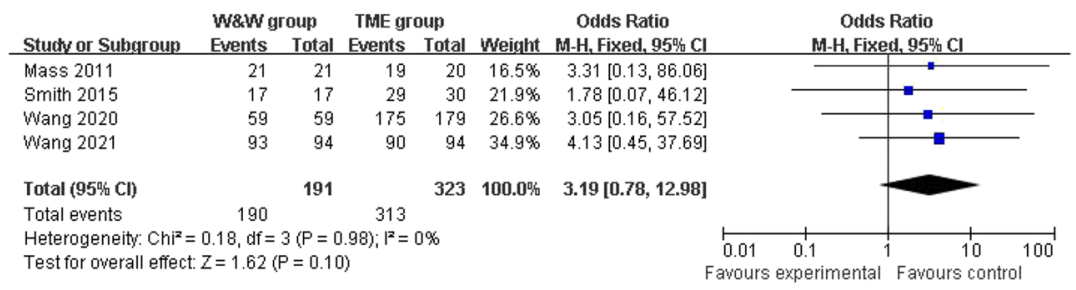

d

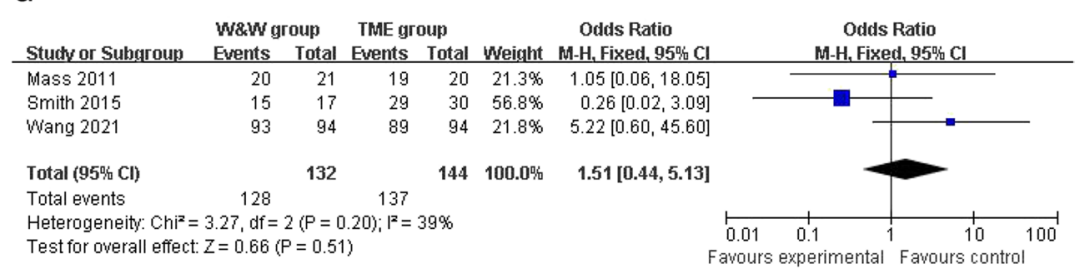

e

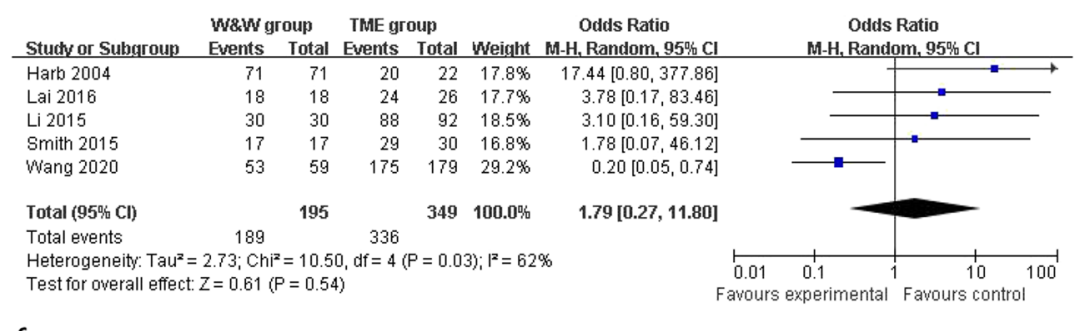

$f$

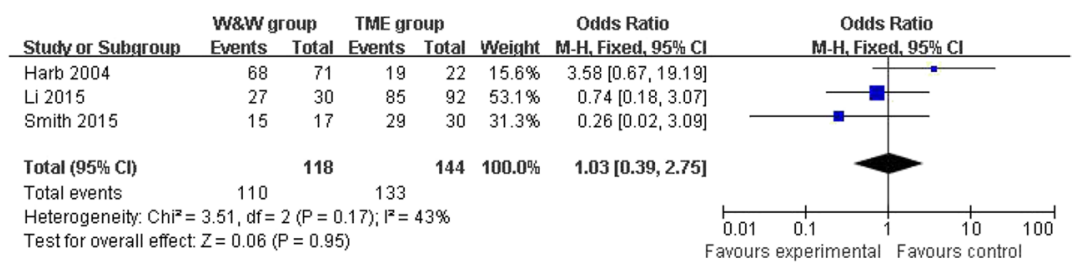

Fig. 3 Outcomes of W\&W group versus TME group. a 2-year DFS. b 2-year OS. c 3-year DFS. d 3-year OS. e 5-year DFS. f 5-year OS 
more details were in the supplementary material 7 [21]. Nowadays, many studies have confirmed the therapeutic effect of the W\&W strategy. TME surgery could bring huge trauma and serious postoperative complications to the patient. We summarized the results of postoperative complications. There was no surgical complication in the W\&W group. But in the TME group, there were hernias in 7 patients, wound infections in 8 patients, intraabdominal abscesses in 7 patients, fistula in 72 patients, and many other complications. Therefore, W\&W strategy has no surgical trauma and can greatly improve the quality of life of patients.

\section{Novelty of the study}

First, compared with the previous articles, the study included the latest studies with consistent baseline data. The results were similar to those of the previous articles (Zhao [31], Dossa [6], and Li [14] study). Second, we excluded studies with significantly different baseline data. Statistical differences in preoperative $\mathrm{T}$ staging, $\mathrm{N}$ staging and TNM staging could cause inconsistent baseline data and affect the final results. Third, the meta-analysis aimed to clarify salvage therapy and provide valuable information for the W\&W strategy.

\section{Comparison with the previous research}

The study of Zhao et al. [31] included 11 studies. He noted that the W\&W group had a higher local recurrence rate than the TME group, and there was no significant difference in other outcomes [26-28, 31, 32]. There were 23 studies in the study of Dossa et al. [6], and they noted that the results (non-regrowth recurrence, cancerspecific mortality, OS, and DFS) were similar in the two groups. The study of Li et al. [14] included nine studies and noted that the non-surgical group had higher 1-, 2-, 3-, and 5-year local recurrence rates than the control groups and the other results were similar in the two groups. Our report further confirmed that the W\&W group had a greater local recurrence rate than the TME group. With the help of physical examination and salvage therapy, W\&W strategy could achieve similar treatment effects as the TME group, and this conclusion was consistent with that of Dattani et al. [33] [33-41]. The local recurrence rate of the W\&W group was $21.6 \%$, and $93 \%$ rectal cancer patients achieved R0 resection after local recurrence. The 3-year OS rate of the W\&W group was 93.5\%. The study of Dattani et al. [33] noted that robust surveillance with suitable salvage surgery makes the W\&W strategy safe and effective. The study of Capelli et al. [42] included nine studies in his study, and he reported that the W\&W strategy can also reduce the rate of colostomy in addition to the above conclusions [42].
The specific details are shown in supplementary material 13.

\section{Outcome results \\ Local recurrence distant metastasis and cancer-related death}

The local recurrence rate in the W\&W group was greater than that in the TME group. We further confirmed the result (high local recurrence rate in the W\&W group) of the study of $\mathrm{Li}$ et al. A total of $86.4 \%$ patients with T3-4 stage disease and $63.9 \%$ patients with $\mathrm{N} 1-2$ stage disease were included before nCRT, and some patients achieved cCR status with negative biopsy after nCRT. However, CCR did not mean no evidence of disease (NED), and some ypT1-3 patients could achieve cCR status. In the TME group, surgery removed the remaining lesions and lymph nodes and reduced the risk of local recurrence $(9.97 \%)$. However, the opposite situation was noted in the W\&W group, the remaining lesions could relapse again and increase the risk of local recurrence. Similar distant metastasis rates and cancer-related deaths of the two groups were also reported by Dossa [6] and Li [14]. Due to the substantial trauma and the postoperative complications of TME group, the W\&W strategy had greatly reduced physical and psychological trauma, and ensured the quality of life while not increasing the probability of DM and CRD rate.

\section{DFS and OS}

The 2-year OS and 2-year DFS in the W\&W group were $100 \%$ and $96.5 \%$ respectively. The 2 -year OS and 2-year DFS in the TME group were $98.4 \%$ and $96.4 \%$ respectively. The 3-year OS, 3-year DFS, 5-year OS and 5-year DFS were similar in the two groups. Initially, we analyzed the studies (including the studies with significant differences in baseline data, such as Lin, Yeom, Lee study) with RevMan 5.0 and 2-DFS in the W\&W group was better than that in the TME group. The specific information is shown in the supplementary material 14 [43]. We hypothesized that the large difference in the baseline data and the incomplete information of Lin, Yeom, Lee, and other studies could affect the results of the research, so we only included studies with consistent baseline data. The similar DFS and OS in the two groups were consistent with previous researches. Patients with cCR status had a good response to NCRT, indicating that the tumor had good biological behavior and that the patients with $\mathrm{CCR}$ status could experience a long survival time in the two groups. Ayloor [16] and Smith [22] reported that the tumor recurrence time was mainly 12 months. Lai [19], Li [20], and Mass [21] reported that the tumor recurrence time was mainly 24 months. We speculated that fewer patients relapsed and did not reach a statistical difference, so the 
DFS was similar in the two groups. With the help of physical examination and salvage surgery, the patient received the corresponding treatment after tumor relapse and the mean overall survival (months) of patients who relapsed also reached more than 50 months. So, the treatment effect of salvage surgery was reliable.

\section{nCRT plan and salvage therapy for local recurrence}

The currently nCRT plan is intensity-modulated radiotherapy (45-50.4 Gy/25-28f) with oral sensitizer (capecitabine $825 \mathrm{mg} / \mathrm{m}^{2} /$ bid po). Surgery could be performed eight weeks after nCRT, and the tumour could be minimized to increase the anus preservation rate and pCR rate. Marit et al. [44] reported that 85 (22.1\%) out of 385 rectal cancer cCR patients relapsed after a median of nine months. Eighty-four (98.8\%) patients underwent salvage surgery, 58 (69\%) patients underwent TME, and $26(30.6 \%)$ patients underwent local excision. The 2-DFS and 2-OS of patients who underwent salvage surgery were $90.3 \%$ and $98.4 \%$ respectively [44]. Irfan [45] et al. proposed that the anastomotic leaks, 30-day morbidity and reintervention rate were similar in the non-deferred surgery group and regrowth deferred surgery group [45]. Simpson [46] et al. noted that the W\&W strategy was safe in this patient cohort, with acceptable rates of local regrowth and survival [46]. In our study, 30 (83.33\%) patients with local recurrence in the W\&W group underwent the salvage therapy. TME surgery was the main type of salvage therapy and could prolong OS. Therefore, with the help of physical examination and salvage treatment, the W\&W strategy could achieve good clinical results with low hospitalization costs [47].

\section{Limitations}

This study might have several limitations. First, no RCTs were available, and the included studies and patients were limited. It could cause bias and affect the results. Second, different levels of medical technology in distinct areas and the partially missing clinical data could affect the final results. Thirdly, the relevant data of adjuvant chemotherapy were incomplete; we were unable to describe the specific plan and treatment effect of adjuvant chemotherapy. It could be an important confounding factor in the TME group following surgery. Finally, minimal information on $\mathrm{T}$ stage and $\mathrm{N}$ stage could lead to limited conclusions regarding specific treatment methods for the patients. Hence, we hope that more RCTs on this topic will emerge in the future.

\section{Conclusion}

The meta-analysis used the latest data to compare the advantages and disadvantages of the W\&W strategy and TME surgery for locally advanced rectal cancer with cCR status after nCRT. The W\&W group had a higher risk of local recurrence than the TME group, but a similar OS was observed in the two groups. With the help of physical examination and salvage treatment, the W\&W strategy could not only decrease surgical trauma and ensure quality of life, but also achieve good clinical results. Hence, the W\&W strategy could represent a beneficial model for rectal cancer with $\mathrm{cCR}$ status.

\section{Abbreviations \\ PNCT: Prospective non-randomized controlled trial; RNCT: Retrospective non-randomized controlled trial; cCR: Complete clinical response; nCRT: Neoadjuvant chemoradiotherapy; 5-FU: 5-Fluorouracil; Cape: Capecitabine; TME: Total mesorectal excision; APR: Abdominal-perineal resection; LAR: Low anterior resection; $\mathrm{Cl}$ : Confidence interval; OR: Odds ratios; OS: Overall survival; SMD: Standardized mean difference.}

\section{Supplementary Information}

The online version contains supplementary material available at https://doi. org/10.1186/s12957-021-02415-y.

\section{Additional file 1.}

Additional file 2. Systematic review.

Additional file 3. PRISMA 2009 Checklist.

Additional file 4. The details of PICOS.

Additional file 5. The information of the search terms.

Additional file 6. The details of neoadjuvant treatment of studies.

Additional file 7. The details of diagnostic criteria (methods) and surveillance strategies of each method in each study.

Additional file 8. Characteristics of the included articles.

Additional file 9. The details of salvage therapy.

Additional file 10. The details of salvage therapy in W\&W group.

Additional file 11. The complications of salvage therapy in W\&W group and TME group.

Additional file 12.

Additional file 13. Comparison between the previous researches.

Additional file 14.

Additional file 15. Editing certificate. A meta-analysis of the watch-andwait strategy versus total mesorectal excision for rectal cancer exhibiting complete clinical response after.

Additional file 16. Editing certificate. A meta-analysis of watch and wait strategy versus total mesorectal excision for rectal cancer with clinical complete response after neoadjuvant chemoradiotherapy.

Additional file 17: Fig 1. Flowchart of the included studies. Fig 2. Outcomes of W\&W group versus TME group. a. local recurrence; b: distant metastasis; c: cancer related death. Fig 3. Outcomes of W\&W group versus TME group. a. 2-year DFS; b: 2-year OS; c: 3-year DFS; d: 3-year OS; e: 5-year DFS; f: 5-year OS

Additional file 18. Statistical method.

Additional file 19. the details of adjuvant chemotherapy of studies.

Additional file 20. Search terms and database. 


\section{Acknowledgements}

We thank the Cancer Hospital of China Medical University, Liaoning Cancer Hospital and Institute for technical assistance. We thank the help of Institute of Process Engineering and Hebei University. We thank the AJE for providing the paper editing service, and the details were shown in supplementary material 15 and 16

\section{Authors' contributions}

All authors participated in the study. XL, ZGJ, and SYM performed the literature search and the acquisition of data. SYM and JQ performed data analysis. WQL and $X L$ participated in the interpretation of data and paper writing. GLY was responsible for the editing, revision, and submission of the paper. All authors read and approved the final version of the article.

\section{Funding}

This work was supported by the Cancer Hospital of China Medical University. The funding project is Natural Science Foundation of Liaoning Province Fund of China (grant nos.2020-MS-060) and Pilot Cancer Research Fund Project of CSCO (Chinese society of clinical oncology; grant nos.Y-2019AZQN-0035) and Dalian University of Technology-Liaoning Cancer Hospital and Institute Cross Joint Fund (grant nos.LD202130).

\section{Declarations}

\section{Ethics approval and consent to participate}

Not applicable

\section{Consent for publication}

Not applicable

\section{Competing interests}

The authors declare that they have no conflicts of interest.

\section{Author details}

${ }^{1}$ Department of General Surgery, Cancer Hospital of China Medical University, Liaoning Cancer Hospital and Institute, No. 44 Xiaoheyan Road, Dadong District, Shenyang 110042, Liaoning Province, People's Republic of China. ${ }^{2}$ School of Life Sciences, Hebei University, Baoding 071002, Hebei Province, People's Republic of China. ${ }^{3}$ Institute of Process Engineering, Chinese Academy of Science, Beijing 100190, People's Republic of China. ${ }^{4}$ Department of Colorectal Surgery, Cancer Hospital of China Medical University, Liaoning Cancer Hospital and Institute, No. 44 Xiaoheyan Road, Dadong District, Shenyang 110042, Liaoning Province, People's Republic of China.

Received: 20 July 2021 Accepted: 30 September 2021

Published online: 18 October 2021

\section{References}

1. Rullier E, Denost Q, Vendrely V, et al. Low rectal cancer: classification and standardization of surgery. Dis Colon Rectum. 2013;56:560-7.

2. Lin H, Wang L, Zhong X, et al. Meta-analysis of neoadjuvant chemotherapy versus neoadjuvant chemoradiotherapy for locally advanced rectal cancer. World J Surg Oncol. 2021;19:141.

3. Gao X, Wang C, Yu Y, et al. Lateral lymph node dissection reduces local recurrence of locally advanced lower rectal cancer in the absence of preoperative neoadjuvant chemoradiotherapy: a systematic review and meta-analysis. World J Surg Oncol. 2020;18:304.

4. Coraglio MF, Eleta MA, Kujaruk MR, et al. Analysis of long-term oncological results of clinical versus pathological responses after neoadjuvant treatment in locally advanced rectal cancer. World J Surg Oncol. 2020;18:313.

5. Dossa F, Acuna SA, Rickles AS, et al. Association between adjuvant chemotherapy and overall survival in patients with rectal cancer and pathological complete response after neoadjuvant chemotherapy and resection. JAMA Oncol. 2018:4:930-7.

6. Benson AB, Venook AP, Al-Hawary MM, et al. Rectal Cancer, Version 2.2018, NCCN Clinical Practice Guidelines in Oncology. J Natl Compr Canc Netw. 2018;16:874-901.
7. Martens MH, Maas M, Heijnen LA, et al. Long-term outcome of an organ preservation program after neoadjuvant treatment for rectal cancer. J Natl Cancer Inst. 2016;108:djw171.

8. Tang B, Lei X, Ai J, et al. Comparison of robotic and laparoscopic rectal cancer surgery: a meta-analysis of randomized controlled trials. World J Surg Oncol. 2021;19:38

9. Timmerman C, Taveras LR, Huerta S. Clinical and molecular diagnosis of pathologic complete response in rectal cancer: an update. Expert Rev Mol Diagn. 2018;18:887-96.

10. Rullier E, Vendrely $V$, Asselineau J, et al. Organ preservation with chemoradiotherapy plus local excision for rectal cancer: 5-year results of the GRECCAR 2 randomised trial. Lancet Gastroenterol Hepatol. 2020;5:465-74.

11. Rullier $E$, Rouanet $P$, Tuech JJ, et al. Organ preservation for rectal cancer (GRECCAR 2): a prospective, randomised, open-label, multicentre, phase 3 trial. Lancet. 2017;390:469-79.

12. Lai IL, You JF, Chern YJ, et al. The risk factors of local recurrence and distant metastasis on $\mathrm{pT} 1 / \mathrm{T} 2 \mathrm{~N} 0$ mid-low rectal cancer after total mesorectal excision. World J Surg Oncol. 2021:19:116.

13. Dossa F, Chesnev TR, Acuna SA, et al. A watch-and-wait approach for locally advanced rectal cancer after a clinical complete response following neoadjuvant chemoradiation: a systematic review and meta-analysis. Lancet Gastroenteol Hepatol. 2017;2:501-13.

14. Li J, Li L, Yang L, et al. Wait-and-see treatment strategies for rectal cancer patients with clinical complete response after neoadjuvant chemoradiotherapy: a systematic review and meta-analysis. Oncotarget. 2016;7:44857-70

15. Downs SH, Black N. The feasibility of creating a checklist for the assessment of the methodological quality both of randomised and nonrandomised studies of health care interventions. J Epidemiol Community Health. 1998;52:377-84.

16. Ayloor Seshadri R, Kondaveeti SS, Jayanand SB, et al. Complete clinical response to neoadjuvant chemoradiation in rectal cancers: can surgery be avoided? Hepatogastroenterology. 2013;60:410-4.

17. Dalton RS, Velineni R, Osborne ME, et al. A single-centre experience of chemoradiotherapy for rectal cancer: is there potential for nonoperative management? Colorectal Dis. 2012;14:567-71.

18. Habr-Gama A, Perez RO, Nadalin W, et al. Operative versus nonoperative treatment for stage 0 distal rectal cancer following chemoradiation therapy: long-term results. Ann Surg. 2004;240:711-7.

19. Lai CL, Lai MJ, Wu CC, et al. Rectal cancer with complete clinical response after neoadjuvant hemoradiotherapy, surgery, or "watch and wait". Int J Colorectal Dis. 2016:31:413-9.

20. Li J, Liu H, Yin J, et al. Wait-and-see or total mesorectal excision for rectal cancer patients with a clinical complete response after neoadjuvant chemoradiotherapy: a cohort study. Oncotarget. 2015;6:4235-61.

21. Maas M, Beets-Tan RG, Lambregts DM, et al. Wait-and-see policy for clinical complete responders after chemoradiation for rectal cancer. J Clin Oncol. 2011;29:4633-40.

22. Smith RK, Fry RD, Mahmoud NN, et al. Surveillance after neoadjuvant therapy in advanced rectal cancer with complete clinical response can have comparable outcomes to total mesorectal excision. Int J Colorectal Dis. 2015;30:769-74.

23. Wang XH, Zhou CJ, Zhang S, et al. Comparison of long-term efficacy between watch and wait strategy and total mesorectal excision in locally advanced rectal cancer patients with clinical complete response after neoadjuvant therapy. Chin J Gastrointest Surg. 2020;23:266-73.

24. Wang QX, Zhang $R$, Xiao WW, et al. The watch-and-wait strategy versus surgical resection for rectal cancer patients with a clinical complete response after neoadjuvant chemoradiotherapy. Radiat Oncol. 2021;16(1):16.

25. Arauio RO, Valadão M, Borges D, et al. Nonoperative management of rectal cancer after chemoradiation opposed to resection after complete clinical response. A comparative study. Eur J Surg Oncol. 2015;41:1456-63.

26. Lee SY, Kim CH, Kim YJ, et al. Oncologic outcomes according to treatment strategy in radiologic complete responders after neoadjuvant chemoradioation for rectal cancer. Oncology. 2015;89:311-8.

27. Renehan AG, Malcomson L, Emsley R, et al. Watch-and-wait approach versus surgical resection after chemoradiotherapy for patients with rectal cancer (theOnCoRe project): a propensity-score matched cohort analysis. Lancet Oncol. 2016:17:174-83. 
28. Smith JD, Ruby JA, Goodman KA, et al. Nonoperative management of rectal cancer with complete clinical response after neoadjuvant therapy. Ann Surg. 2012;256:965-72.

29. Lin GP, Lee KD, Wang JY, et al. Status for clinically complete remission rectal cancer after concomitant chemo-radiotherapy in Taiwan. Asian J Surg. 2018;41:203-6.

30. Habr-Gama A, São Julião GP, Vailati BB, et al. Organ preservation in cT2N0 rectal cancer after neoadjuvant chemoradiation therapy: the impact of radiation therapy dose-escalation and consolidation chemotherapy. Ann Surg. 2019;269:102-7.

31. Zhao GH, Deng L, Ye DM, et al. Efficacy and safety of watch and wait strategy versus total mesorectal excision and local excision for rectal cancer with cCR response after neoadjuvant chemoradiotherapy: a metaanalysis. World J Surg Oncol. 2020;18:232.

32. Yeom SS, Lee SY, Kim CH, et al. Non-operative treatment outcome for rectal cancer patient with clinical complete response after neoadjuvant chemoradiotherapy. Asian J Surg. 2019;42:823-31.

33. Dattani M, Heald RJ, Goussous G, et al. Oncological and survival outcomes in watch and wait patients with a clinical complete response after neoadjuvant chemoradiotherapy for rectal cancer. Ann Surg. 2018;268:955-67.

34. Nakagawa WT, Rossi BM, de O Ferreira F, et al. Chemoradiation instead of surgery to treat mid and low rectal tumors: is it safe? Ann Surg Oncol. 2002;9(6):568-73.

35. Habr-Gama A, Gama-Rodrigues J, São Julião GP, et al. Local recurrence after complete clinical response and watch and wait in rectal cancer after neoadjuvant chemoradiation: impact of salvage therapy on local disease control. Int J Radiat Oncol Biol Phys. 2014;88(4):822-8.

36. Perez RO, Habr-Gama A, São Julião GP, et al. Predicting complete response to neoadjuvant CRT for distal rectal cancer using sequential PET/CT imaging. Tech Coloproctol. 2014;18(8):699-708.

37. Appelt AL, Pløen J, Harling H, et al. High-dose chemoradiotherapy and watchful waiting for distal rectal cancer: a prospective observational study. Lancet Oncol. 2015;16(8):919-27.

38. Sanchez Loria F, Iseas S, O'Connor JM, et al. Non-surgical management of rectal cancer. Series of 68 cases, long follow up in two leading centres in Argentina. Dig Liver Dis. 2016;48(11):1372-7.
39. Vaccaro CA, Yazyi FJ, Ojra Quintana G, et al. Locally advanced rectal cancer: preliminary results of rectal preservation after neoadjuvant chemoradiotherapy. Cir Esp. 2016;94(5):274-9.

40. Kusters M, Slater A, Betts M, et al. The treatment of all MRI-defined low rectal cancers in a single expert centre over a 5-year period: is there room for improvement? Colorectal Dis. 2016;18(11):0397-404.

41. Creavin B, Ryan E, Martin ST, et al. Organ preservation with local excision or active surveillance following chemoradiotherapy for rectal cancer. $\mathrm{Br}$ J Cancer. 2017;116(2):169-74.

42. Capelli G, De Simone I, Spolverato G, et al. Non-operative management versus total mesorectal excision for locally advanced rectal cancer with clinical complete response after neoadjuvant chemoradiotherapy: a GRADE Approach by the Rectal Cancer Guidelines Writing Group of the Italian Association of Medical Oncology (AIOM). J Gastrointest Surg. 2020;24(9):2150-9.

43. Dapper H, Oechsner M, Münch S, et al. Dosimetric analysis and comparison of reduced longitudinal cranial margins of VMAT-IMRT of rectal cancer. Radiat Oncol. 2018;13:169.

44. Van der SME, Figueiredo N, Beets GL. Management and outcome of local regrowths in a watch-and wait prospective cohort for complete responses in rectal cancer. Ann Surg. 2020;7 (Online ahead of print).

45. Nasir I, Fernandez L, Vieira P, et al. Salvage surgery for local regrowths in Watch \& Wait - Are we harming our patients by deferring the surgery? Eur J Surg Oncol. 2019;45:1559-66.

46. Simpson G, Hopley P, Wilson J, et al. Long-term outcomes of real world 'watch and wait' data for rectal cancer after neoadjuvant chemoradiotherapy. Colorectal Dis. 2020;22:1568-76.

47. Miller JA, Wang H, Chang DT, et al. Cost-effectiveness and qualityadjusted survival of watch and wait after complete response to chemoradiotherapy for rectal cancer. J Natl Cancer Inst. 2020;112:792-801.

\section{Publisher's Note}

Springer Nature remains neutral with regard to jurisdictional claims in published maps and institutional affiliations.
Ready to submit your research? Choose BMC and benefit from:

- fast, convenient online submission

- thorough peer review by experienced researchers in your field

- rapid publication on acceptance

- support for research data, including large and complex data types

- gold Open Access which fosters wider collaboration and increased citations

- maximum visibility for your research: over $100 \mathrm{M}$ website views per year

At BMC, research is always in progress.

Learn more biomedcentral.com/submissions 